\title{
Concept formation as a function of perceptual pretraining and knowledge of results'
}

BILL R. BROWN, DON W. WALKER, ${ }^{2}$ and SELBY $H$. EVANS, Texas Christian University, Fort Worth, Tex. 76129

The present study assessed the joint effects of perceptual pretraining $(P T)$ and knowledge of results $(K R)$ on performance in a task requiring discrimination among stimuli sampled from three different schema families. Neither schematic concept formation (SCF) nor didactic concept formation was significantly influenced by increasing the amount of pretraining. Didactic concept formation occurred if $K R$ was provided, but the low level of stimulus redundancy in the task prevented the occurrence of SCF.

Schema theory (Attneave, 1957; Oldfield, 1954) suggests that the encoding of the schematic aspects of stimuli reduces information processing and storage requirements. Specific instances could be efficiently stored in the form of schema plus correction (Woodworth, 1938). The schema rule is assumed to be abstracted as a set of commonly occurring characteristics in a collection of otherwise different schema families. Thus, schema theory clearly requires extension if it is to deal adequately with ordinary human perception.

The following suppositions about the environment and about perceptual processes extend schema theory to the mixed schemata case: (1) A major component of environmental orderliness is that objects can be identified as belonging to categories. There is the implication of natural categories and these are defined by the orderliness of the environment (Evans, 1968). (2) Each schema family corresponds to an equivalence class or statistical concept. Each 'concept has a number of attributes associated with it. Assignment of objects to a class is based on a large number of attributes which have some statistical association with the class. Collectively these attributes are sufficiently reliable to permit assignment of objects to the correct category with a small probability of error (Evans, 1967a). (3) The information necessary for the. selection of relevant attributes and for the construction of assignment rules is provided by the clusters of covarying characteristics associated with schema families (Evans, 1967a).

Schematic concept formation has been defined by Evans as "the development of the ability to assign objects to their corresponding schema families on the basis of the information derived from perceiving the objects, without any other source of information as to the appropriate categorization, and without prior familiarization with the relevant schema [1967a, p. 88]." In traditional concept formation, the necessary information for categorization is acquired through knowledge of results. Such concepts may be termed didactic concepts; they need not be associated with schemata. Schematic concept formation has been demonstrated in several different tasks (Brown, Walker, \& Evans, 1968; Edmonds, Mueller, \& Evans, 1966; Evans \& Arnoult, 1967).

The SCF process requires overdetermined equivalence classes. An overdetermined equivalance class or concept is one which has more attributes associated with it than are needed for near-perfect classification. Constraint redundancy $(R c)$ is a measure of overdetermination. The Rc measure has been discussed in detail by Evans (1967b) and represents the extent to which a population of stimuli adhere to a schema. Low levels of Rc prevent the occurrence of SCF (Brown, Walker, \& Evans, 1968).

Perceptual pretraining (Tighe \& Tighe, 1968) requires Ss to make nonreinforced judgments of successively presented stimuli which vary along the dimensions appearing in a subsequent discrimination task. The SCF process implies that concepts will be formed without any instructions to form categories. Hence, PT procedures which provide the opportunity to inspect stimuli of different schema families should permit the formation of schematic concepts; the degree to which SCF has occurred would be demonstrable by a subsequent shift to a test task requiring the assignment of the stimuli to their corresponding schema families without $K R$. The presence of $K R$ during the test task permits didactic concept formation and thus might be expected to minimize any facilitory effects attributable to pretraining.

This study was intended to achieve the following objectives: (1) To compare the effects of KR and no-KR (NKR) upon concept learning with low Rc stimuli; and (2) to assess the effects of differing amounts of PT on SCF and didactic concept formation with low Rc stimuli. The following hypotheses were investigated: (1) With no PT, KR facilitates performance in comparison to the NKR condition; and (2) increasing the amount of PT facilitates the SCF process; the presence of KR permits didactic concept learning and thus obscures the facilitating effect of pretraining.

\section{METHOD}

The Ss were 60 naive undergraduates enrolled in psychology courses at Texas Christian University.

The VARGUS 7 computer program (Evans, 1967c) was used to generate $40 \% \mathrm{Rc}$ histoform patterns consisting of three schema families.

The Ss were given 20 trials with no KR by means of Multilithed booklets. There were three levels of pretraining. The zero-PT groups received 20 placebo trials in which each $S$ made area judgments of geometric figures (circles, triangles, and squares). The 10-trial PT groups received 10 placebo trials and then 10 trials in which each $S$ made area judgments of the VARGUS 7 patterns. The 20-trial PT groups received 20 trials in which each $S$ made area judgments of the VARGUS 7 patterns. For all groups an area judgment consisted of merely judging which of three stimuli had the largest area. Consequently, the Ss receiving 10 and 20 PT trials had the opportunity to inspect patterns of three schema families without instructions to form categories. The Ss were given 20 sec for each trial.

Following the PT trials the Ss were given 20 test trials by means of Multilithed booklets. The patterns were different instances, but of the same schema families from which the PT patterns were sampled. A typical test trial consisted of viewing a single pattern of a schema family for $10 \mathrm{sec}$, followed by the attempted selection of a pattern from the same schema family from a group of three patterns of different schemata. The Ss were instructed to select the pattern most similar to the single pattern previously studied. Each $S$ was allowed 20 sec to make a selection. The $\mathrm{KR}$ groups were then shown the correct answer for $10 \mathrm{sec}$; the NKR groups viewed a blank page during this interval. Precautions were taken to insure that the Ss receiving $K R$ could not view the correct answer until a selection had been made.

\section{RESULTS AND DISCUSSION}

A two-way analysis of variance was applied to the performance data; the dependent variable was the number of correct choices for all 20 trials. The KR main effect was significant ( $F=9.46, \mathrm{df}=1 / 54, \mathrm{p}<.01$ ). Inspection of Fig. 1 shows that performance was higher under $K R$ than under the NKR condition. Neither the PT main effect nor the PT by KR interaction was significant. 


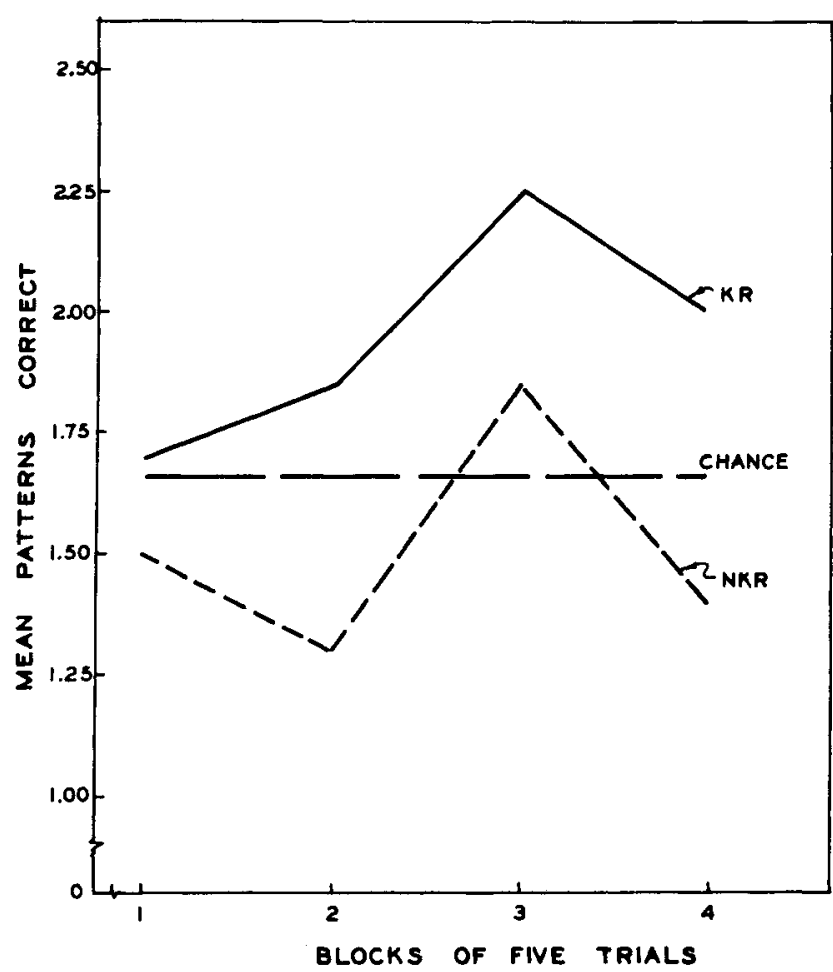

Fig. 1. Mean number of correct responses for blocks of five trials for KR and NKR groups, both averaged over levels of pretraining.

The results support the supposition that SCF requires overdetermined categories and that low levels of Rc prevent the SCF process from occurring. Unless the stimuli are sufficiently redundant, the information necessary for the selection of relevant attributes and the construction of assignment rules cannot be extracted from the stimuli themselves without external reinforcement. It is not clear, however, what level of Rc is sufficient for SCF to occur. Several studies (Brown, 1968; Evans \& Arnoult, 1967) have demonstrated SCF in tasks using 60 trials with 50\%-Rc stimuli. Although it is likely that the stimuli must at least approach the $50 \%-\mathrm{Rc}$ level for humans to exhibit SCF, a task using a large number of trials with $40 \%-\mathrm{Rc}$ stimuli requires investigation.

Didactic concept formation, on the other hand, occurs with a low level of Rc. Under all PT conditions, the KR groups performed at a higher level than did the NKR groups. This result is consistent with the first hypothesis and supports the distinction between SCF and didactic concept formation made by Evans (1967a). Previous experiments (Brown, Walker, \& Evans, 1968; Edmonds, Mueller, \& Evans, 1966; Evans \& Edmonds, 1966; Wright \& Dixon, 1968), however, have shown that the administration of KR under high Rc conditions does not necessarily facilitate performance in that the overdetermined stimuli permit the occurrence of SCF.
No significant effect of PT was observed in this experiment. Inspection of the data, however, showed that the facilitory effect of KR was the largest under the 10- and 20-trial PT conditions. This observation is contrary to the second hypothesis and suggests that more effective PT procedures, e.g., stimulus reproduction, may enhance performance under low $R c$ conditions when $\mathrm{KR}$ is administered.

The failure of PT to facilitate performance under the NKR condition (in comparison to zero-PT) was also contrary to the second hypothesis. Edmonds \& Mueller (1968) found that area PT facilitated schema learning in a single-schema case in which the stimuli were $67 \%$ Rc. There is thus the possibility that PT may improve the level of SCF if the stimuli are sufficiently redundant. Further investigations of the SCF process using other PT procedures and different levels of $\mathrm{Rc}$ are clearly required.

\section{REFERENCES}

ATTNEAVE, F. Transfer of experience with a class-schema to identification-learning of patterns and shapes. Journal of Experimental Psychology, 1957, 54, 81-88.

BROWN, B. R. A model for schematic concept formation: Further development and assessment. Unpublished doctoral dissertation, Texas Christian University, 1968.

BROWN, B. R., WALKER, D. W., \& EVANS, S. H. Schematic concept formation as a function of constraint redundancy and knowledge of results. Psychonomic Science, 1968, 11, 75-76.

EDMONDS, E. M., \& MUELLER, M. R. Effects of incidental training and reinforcement on mixed schema learning. Psychonomic Science, $1968,10,75-76$.

EDMONDS, E. M., MUELLER, M. R., \& EVANS, S. H. Effects of knowledge of results on mixed schema discrimination. Psychonomic Science, 1966, 6, 377-378.

EVANS, S. H. A brief statement of schema theory. Psychonomic Science, 1967a, 8, 87-88.

EVANS, S. H. Redundancy as a variable in pattern perception. Psychological Bulletin, 1967b, 67, 104-113.

EVANS, S. H. VARGUS 7: Computed patterns from Markov processes. Behavioral Science, $1967 \mathrm{c}, 12,323-328$.

EVANS, S. H. The conceptual basis of schema theory. Symposium paper presented at Southwestern Psychological Association, April, 1968.

EVANS, S. H., \& ARNOULT, M. D. Schematic concept formation: Demonstration in a free sorting task. Psychonomic Science, 1967, 9, 221-222.

EVANS, S. H., \& EDMONDS, E. M. Schema discrimination as a function of training. Psychonomic Science, 1966, 5, 303-304.

OLDFIELD, R. C. Memory mechanisms and the theory of schemata. British Journal of Psychology, 1954, 45, 14-23.

TIGHE, L. S., \& TIGHE, T. J. Transfer from perceptual pretraining as a function of number of stimulus values per dimension. Psychonomic Science, 1968, 12, 135-136.

WOODWORTH, R. S. Experimental psychology. New York: Holt, 1938. WRIGHT, A. D., \& DIXON, T. R. Knowledge of results in schematic concept formation. Human Resources Research Office Professional Paper 17-68, The George Washington University, Alexandria, Virginia, 1968.

\section{NOTES}

1. This research was supported in part by a Department of Defense Project THEMIS (DAADO5-68-C-0176) under the Department of the Army and by Grant No. PS-6672 from the TCU Research Foundation.

2. Now at Medical Research Services, Veterns Administration Hospital, Gainesville, Florida. 